HNO

1998 $\cdot$ 46:303-310 @ Springer-Verlag 1998

Redaktion

H.P.Zenner, Tübingen
Übersicht

J.C. Türp - Department of Biologic and Materials Sciences (Direktor:Prof.Dr.C.S.Stohler),

School of Dentistry, The University of Michigan, Ann Arbor, Michigan, USA

\section{Zusammenfassung}

In der (zahn)medizinischen Literatur wird seit langem über einen möglichen Zusammenhang zwischen Myoarthropathien (MAP) des Kausystems und Otalgien bzw. Tinnitus gemutmaßt.Ziel dieses Beitrags ist es, den aktuellen Stand zu dieser Frage darzustellen. Eine Analyse relevanter, zwischen 1947 und 1996 veröffentlichter Studien zeigt, daß in dem überwiegenden Teil der Untersuchungen von einer relativ hohen Prävalenz von Ohrenschmerzen bzw. Ohrgeräuschen bei MAP-Patienten berichtet wurde. Eine kausale Beziehung konnte bislang allerdings nicht hergestellt werden. Auch bleiben Erklärungsversuche, die das gleichzeitige Auftreten von MAP- und Ohrsymptomen, vor allem Ohrgeräuschen, auf eine gemeinsame Ursache zurückführen, spekulativ. Zu berücksichtigen ist, daß ein zufälliges Zusammentreffen von MAP- und auralen Symptomen aufgrund ihrer weiten Verbreitung möglich ist. Ein methodischer Schwachpunkt der meisten Studien besteht in der Nichtberücksichtigung von Vergleichsgruppen. Behauptungen zur Wirksamkeit bestimmter zahnärztlicher Maßnahmen für die Behandlung von Otalgien und Tinnitus bei MAP-Patienten finden angesichts fehlender prospektiver, kontrollierter und randomisierter Untersuchungen bislang wenig Unterstützung.

\section{Schlüsselwörter}

Kiefergelenke · Kaumuskulatur . Myoarthropathien · Ohrenbeschwerden . Lig. discomalleolare

\title{
Zum Zusammenhang zwischen Myoarthropathien des Kausystems und Ohrenbeschwerden (Otalgie, Tinnitus)
}

1 on (MAP) des Kausystems geschilderte Beschwerden beschränken sich in vielen Fällen nicht auf die Kiefergelenke und Kaumuskulatur. Neben Kopfschmerzen werden vor allem Ohrenschmerzen und Ohrgeräusche relativ häufig genannt [ 7 , 35]. Obwohl sich die (zahn)medizinische Fachliteratur bereits seit vielen Jahrzehnten mit der Beziehung zwischen Ohrenbeschwerden und Beschwerden im Kausystem beschäftigt $[3-5,7,8,13,15,18,19,25,35,50,51]$, sind die gewonnenen Erkenntnisse beschränkt und die Empfehlungen für den Praktiker widersprüchlich. Die Hauptschwierigkeit besteht darin, herauszufinden, $o b$ von MAP-Patienten angegebene Ohrenbeschwerden eine Folge der bestehenden MAP sind oder unabhängig von den MAP vorkommen. Ziel des vorliegenden Beitrags ist es, nach einer kurzen Einführung in den Themenkomplex der MAP des Kausystems den heutigen Wissensstand zum Zusammenhang zwischen MAP und den beiden am häufigsten angegebenen Ohrenbeschwerden, Otalgie und Tinnitus, darzustellen.

\section{Myoarthropathien des Kausystems}

Myoarthropathien des Kausystems sind durch Schmerzen im Bereich der Kiefergelenke und der Kaumuskeln (bzw. deren Sehnen) und Einschränkungen der Unterkieferbeweglichkeit gekennzeichnet [31]. Traditionell werden auch
Kiefergelenkgeräusche (Knacken; Reiben) als typische MAP-Symptome angeführt; ihre klinische und pathologische Bedeutung wurde in letzter Zeit jedoch vermehrt in Frage gestellt [48]. Die Bezeichnung "Myoarthropathie“ selbst ist ein Oberbegriff, der verschiedene, als pathologisch gewertete Erscheinungen im Bereich der Kiefergelenke und Kaumuskulatur umfaßt. Klinisch werden derzeit eine Vielzahl miteinander konkurrierender Klassifikationssysteme verwendet [30], was die Kommunikation unter Behandlern bisweilen erschwert.

Alternativ $\mathrm{zu}$ der Bezeichnung „Myoarthropathie“ wird in der deutschsprachigen zahnärztlichen Literatur oft der Begriff „Funktionsstörung“ gebraucht. Schulte, der den Ausdruck "Myoarthropathie“ in die zahnmedizinische Nomenklatur eingeführt hatte, wies jedoch bereits Anfang der 8oer Jahre darauf hin, daß beide Begriffe deutlich zu unterscheiden sind: „Zeichen der Funktionsstörung sind im stomatognathen System sehr viel häufiger nachweisbar als Beschwerden bzw. subjektive krankhafte Befunde. Die Begriffe Myoarthropathie, Schmerzdysfunktionssyndrom etc. sollten deshalb (griech. Pathos=das Leiden) nur dann benutzt werden, wenn tatsächlich subjektive krankhafte Befunde bestehen“ [38]. Lei-

Dr.J.C.Türp, Visiting Assistant Professor Department of Biologic and Materials Sciences, School of Dentistry, The University of Michigan, Ann Arbor, MI 48109-1078, USA 
HNO

1998 • 46:303-310 ๔ Springer-Verlag 1998

\section{J.C.Türp}

\section{The association between tempo- romandibular disorders and ear symptoms of otalgia and tinnitus}

\section{Summary}

Speculations concerning a possible association between temporomandibular disorders (TMDs) and ear symptoms of otalgia and tinnitus have been made in the dental and medical literature for a long time. Current understanding of this association is reviewed. An analysis of relevant studies published between 1947 and 1996 shows that a relatively high percentage of TMD patients reported the presence of ear pain and noise. However, a cause-effect relationship has not been established to date. Theories that try to explain the concurrence of TMD symptoms and ear symptoms (particularly tinnitus) by a common underlying cause remain speculative. It has to be taken into consideration that due to their high prevalence, TMDs and ear symptoms in a given patient may be present by chance alone. A methodological weakness of most of the studies available is the fact that control groups were not considered. Owing to the lack of prospective, controlled, and randomized studies, statements regarding the effectiveness of certain dental measures for the treatment of otalgia and tinnitus in TMD patients are not well supported.

\section{Key words}

Temporomandibular joint . Masticatory muscles · Ear symptoms . Discomalleolar ligament

\section{Übersicht}

der wird in der Literatur eine solche Differenzierung kaum getroffen. Stattdessen trifft man eine Vielzahl von Ausdrücken an, von denen ein großer Teil, darunter die Bezeichnung „Costen-Syndrom" sowie der aus dem Amerikanischen („myofascial pain dysfunction syndrome“) abgeleitete Begriff „myofasziales (Schmerzdysfunktions-) Syndrom", heute allerdings kaum mehr gebräuchlich sind.

Epidemiologische Studien haben gezeigt, daß in der Bevölkerung bei 40-75\% aller Erwachsenen mindestens ein „objektives“, d.h. von einem Untersucher feststellbares bzw. auslösbares Symptom einer Funktionsstörung des Kausystems (z.B. Schmerzempfindlichkeit bei Palpation von Kaumuskeln) festgestellt werden kann, während subjektive, d.h. vom Patienten berichtete Symptome (z.B. Kiefergelenkschmerzen, Kiefergelenkknacken) bei rund $1 / 3$ der Befragten nachweisbar sind [31]. Die Zahl der in den USA lebenden Erwachsenen mit persistierenden Schmerzen im Bereich der Kaumuskulatur oder Kiefergelenke wird von der amerikanischen Gesundheitsbehörde National Institutes of Health (NIH) auf rund 7 Mio. geschätzt [43].

Im Laufe der Jahrzehnte wurden unterschiedliche Ätiologiemodelle für die Entstehung von MAP vorgeschlagen, darunter eine Verlagerung des Condylus mandibulae nach retral, eine ,nicht-harmonische" neuromuskuläre Position des Unterkiefers relativ zum Oberkiefer, Zahnvorkontakte (Interferenzen), Knirschen bzw. Pressen der Zähne (Bruxismus) und psychologische Faktoren (s. Übersicht bei [24]). Von den meisten Zahnmedizinern wurden dabei Abweichungen von einer „idealen“ Okklusion der Zähne traditionell eine große, wenn nicht ausschlaggebende Bedeutung eingeräumt. In jüngster Zeit ist allerdings deutlich geworden, daß okklusalen Faktoren eine weitaus weniger gewichtige Rolle in der Ätiologie der MAP zukommt, als man in der Vergangenheit angenommen hatte $[40,41]$. Auch der vielfach behauptete Zusammenhang zwischen Bruxismus und MAP ist noch längst nicht bewiesen [22]. Die Tatsache, daß Lehrbücher heute meist von einer „multifaktoriellen Ätiologie“ sprechen, darf nicht darüber hinwegtäuschen, daß die Kenntnisse über die genauen Mechanismen der Entstehung von MAP noch äußerst beschränkt sind. Aus diesem Grunde ist eine Untergliederung der MAP in unterschiedliche Krankheitsbilder bislang lediglich im Hinblick auf die An- oder Abwesenheit bestimmter Symptome möglich. Die entsprechenden Einteilungskriterien variieren zwischen den verschiedenen Klassifikationssystemen bisweilen deutlich.

Differentialdiagnostisch müssen Schmerzzustände ausgeschlossen werden, die ähnliche Symptome hervorrufen, wie sie bei MAP auftreten. Dazu zählen u.a. Trigeminusneuralgie und atypischer Gesichtsschmerz [46], Spannungskopfschmerzen, Otitis media und Tumoren. Bei Verdacht auf ein Geschehen außerhalb des Kausystems ist eine Überweisung an einen entsprechenden medizinischen Fachkollegen empfehlenswert.

Die vorhandenen Wissensdefizite hinsichtlich der Ätiologie der MAP bedingen, daß eine spezifische Therapie der Beschwerden bislang nicht möglich ist. Im Mittelpunkt der Behandlungsstrategien steht die symptomatische Reduktion von Schmerzen sowie die Verbesserung einer eingeschränkten Unterkieferbeweglichkeit. Bei der Wahl geeigneter Behandlungsmittel stehen konservative, reversible Mittel, wie z.B. intraorale Okklusionsschienen, Physiotherapie, Entspannungsverfahren und der gezielte Einsatz von Pharmaka, gegenüber irreversiblen und invasiven Maßnahmen (wie z.B. offene chirurgische Maßnahmen an den Kiefergelenken) im Vordergund [28, 45]. Kieferorthopädische Behandlungen mit dem Ziel der Prävention oder Therapie von MAP werden demgegenüber nicht empfohlen [23]. Diese Auffassung kam auch im Schlußdokument einer im Jahre 1996 ausgerichteten, NIH-gesponsorten internationalen Konsensuskonferenz zur Diagnose und Therapie der MAP des Kausystems (NIH Technology Assessment Conference on the Management of Temporomandibular Disorders, 29. April-1. Mai 1996, Bethesda, Maryland) zum Ausdruck [28]. Ein aus Klinikern und Forschern verschiedener wissenschaftlicher Disziplinen bestehendes Expertengremium stellte darin unter anderem fest, daß bisher keine überzeugenden Informationen vorliegen, daß kieferorthopädische Behandlung dem Auftreten von MAP des Kausystems vorbeugt [28]. 
Tabelle 1

Auswahl von Studien zur Prävalenz von Otalgie und Tinnitus bei Patienten mit MAP des Kausystems. Nur Studien mit einer großen Patientenzahl $(n \geq 90)$ sind berücksichtigt

\begin{tabular}{lccc} 
Autor & $\begin{array}{l}\text { Gesamtzahl der } \\
\text { MAP-Patienten }\end{array}$ & $\begin{array}{l}\text { Anzahl der } \\
\text { MAP-Patienten } \\
\text { mit Otalgie [n (\%)] }\end{array}$ & $\begin{array}{l}\text { Anzahl der } \\
\text { MAP-Patienten } \\
\text { mit Tinnitus [n (\%)] }\end{array}$ \\
\hline Goodfriend [13] & 168 & & $(14)$ \\
Hankey [15] & 100 & & $(1)$ \\
Vero [50] & 150 & $64(42)$ & $2(2)$ \\
Gelb et al.[11] & 742 & $263(35)$ & $311(42)$ \\
Myrhaug [27] & 1986 & $444(22)$ & \\
Posselt [32] & 731 & & $($ ca.4) \\
Morgan [25] & 90 & & $60(67)$ \\
Carlsson et al.[5] & 350 & & $(15)$ \\
Gelb et al.[10] & 200 & & $71(35,5)$ \\
Wedel und Carlsson [51] & 350 & $86(82)$ & $8(2)$ \\
Bush [4] & 105 & & $35(33)$ \\
Rubinstein et al.[35] & 376 & & $93(25)$ \\
Chole und Parker [7] & 338 & & $(59)$ \\
Kempf et al.[19] & 138 & $167(42)$ & $18(13)$ \\
Keersmaekers et al. [18] & 400 & &
\end{tabular}

Nur am Rande sei darauf hingewiesen, daß bei Patienten mit persistierenden MAP einer psychologischen Schmerzdiagnostik und der Integration psychologischer Therapiemaßnahmen in ein Behandlungsprogramm große Bedeutung beigemessen wird. Der Grund dafür liegt in der allgemeinen Akzeptanz eines biopsychosozialen Schmerzkonzepts, wonach somatische, subjektiv-psychologische, psychophysiologische, verhaltensbezogene und soziale Faktoren gemeinsam an der Entstehung und Aufrechterhaltung des Schmerzerlebens beteiligt sind [45].

\section{MAP und Ohrenschmerzen}

Berichte in medizinischen Fachzeitschriften über das gleichzeitige Vorhandensein von Kiefergelenkbeschwerden und Ohrenschmerzen reichen bis in das 19. Jahrhundert zurück. Die erste diesbezügliche Veröffentlichung stammt aus dem Jahre 1895. Der Berliner Assistenzarzt Alfred Bruck [3] stellte darin die Fälle von 5 Patientinnen vor, die neben Kiefergelenkschmerzen über Otalgien klagten. Als Ursache für die im $\mathrm{Ohr}$ wahrgenommenen und bei Bewegungen des Unterkiefers an Intensität zunehmenden Schmerzen sah Bruck eine Irritation der „Rr. articulares des N. auriculo-temporalis bis in dessen Ohräste" an. Diese Hypothese stellte in den nachfolgenden Jahrzehnten die am häufigsten genannte Erklärung für eine bei Kiefergelenkschmerzen vorhandene Otalgie dar [8]. Ende der 4oer Jahre wurde von anatomischer Seite der Einwand hervorgebracht, daß der N. auriculotemporalis aufgrund seines Verlaufs entlang des Collum mandibulae kaudal der Insertion der Kiefergelenkkapsel und kaudal der Pars tympanica selbst bei einer forcierten Rückwärtsverlagerung des Kondylus nicht komprimiert werden kann [42], wie von Costen [8] und anderen behauptet worden war. Nach neueren Untersuchungen von Johansson et al. [17] scheint es allerdings denkbar, daß unter bestimmten Voraussetzungen, nämlich bei einem medial positionierten Discus articularis, eine mechanische Irritation eines von seiner normalen topographischen Lage abweichenden N. auriculotemporalis (und benachbarter Nerven) möglich ist.

Angaben zur Prävalenz von Ohrenschmerzen bei MAP-Patienten variieren von Autor zu Autor. Die in Tabelle 1 zusammengefaßten Untersuchungen lassen allerdings erkennen, daß Otalgien bei MAP-Patienten weit verbreitet sind. Im Vergleich dazu berichteten Gross et al. [14], daß von 1109 Patienten, die zu einem routinemäßigen Zahnarztbesuch erschienen waren, aber keine MAP-Beschwerden aufwiesen, lediglich
64 Personen (5,8\%) angaben, am Tage unter Ohrenschmerzen zu leiden, während nur 25 Patienten (2,3\%) von einer nächtlichen Otalgie betroffen waren.

Die Ergebnisse einer kürzlich veröffentlichten kontrollierten Studie zeigten, daß nach Abschluß einer MAP-Behandlung ein Großteil der Patienten eine Besserung ihrer Otalgien angab, obwohl sich die angewandte Therapie ausschließlich auf die MAP-Symptome konzentrierte. Keersmaekers et al. [18] verglichen 233 MAP-Patienten ohne Ohrsymptome (Durchschnittsalter: 36 Jahre) mit 167 MAP-Patienten (Durchschnittsalter: 39,4 Jahre), die unter Ohrenschmerzen litten. Der HNO-Befund in letzterer Gruppe war negativ; eine Therapie für die Ohrenbeschwerden war nicht eingeleitet worden. Bei der Erstuntersuchung wurde die Gesamtintensität der Schmerzen (d.h. der muskuloskeletalen Gesichtsschmerzen sowie, falls vorhanden, der Ohrenschmerzen) von den meisten Patienten als mittelstark bezeichnet ( $78 \%$ der Patienten ohne bzw. $76 \%$ der Patienten mit Otalgie); $13 \%$ der Patienten ohne Otalgie gaben starke Schmerzen an, gegenüber $21 \%$ in der Gruppe mit Ohrenbeschwerden; dieser Unterschied war statistisch signifikant. Bei beiden Patientengruppen wurden anschließend die gleichen, ausschließlich auf die MAP gerichteten Behandlungsmaßnahmen durchgeführt (Beratung, intraorale Okklusionsschienen, Physiotherapie, sowie in einigen Fällen Gabe nicht-steroidaler Analgetika). Bei der ein Jahr später erfolgten Nachuntersuchung wies der Großteil der Patienten keine oder lediglich gering ausgeprägte Schmerzen auf (84 bzw. $66 \%$ in den jeweiligen Gruppen). Starke Schmerzen waren nur noch bei 2 bzw. 3\% der Patienten vorhanden; 48\% der Patienten mit Otalgie zu Beginn der Studie hatten keine Ohrenschmerzen mehr, bei weiteren $32 \%$ waren die Otalgien gering ausgeprägt oder traten nur gelegentlich auf. Die Autoren selbst schlossen aus ihren Untersuchungsergebnissen, daß MAP-Patienten mit Otalgien ebenso gut auf konservative Behandlungsmaßnahmen ansprechen wie MAP-Patienten ohne Ohrenschmerzen [18]. Ob jedoch der deutliche Rückgang der Otalgien auf spezifische Wirkungen der primär auf die MAP ausgerichteten Behandlungsmaßnahmen zurückzuführen ist, geht aus der Untersuchung 


\section{Übersicht}

nicht hervor. Es ist ebenso möglich, daß die eingetretene Besserung aufgrund unspezifischer Effekte (Plazeboeffekte) zustande kam (wie beispielsweise durch die mit der Therapie verbundene Erwartungshaltung der Patienten und des Behandlers) oder aber Ausdruck einer spontanen Remission oder des natürlichen Verlaufs der Beschwerden ist. Der Einfluß von nicht-spezifischen Effekten wird bei einem nach eingeleiteter Therapie beobachteten Schmerzrückgang oftmals unterschätzt, so daß eine eingetretene Besserung der Beschwerden oft zu Unrecht einer spezifischen Wirkung des eingesetzten Behandlungsmittels zugeschrieben wird [44].

\section{MAP und Tinnitus}

Angaben zur Verbreitung von Tinnitus in der Bevölkerung schwanken je nach Studie beträchtlich. In der Literatur werden Werte zwischen 2,3 und 44,5\% genannt, mit einem Median zwischen 15 und 20\% [34]. Nach Angaben der "American Tinnitus Association" leiden in den USA rund 40 Mio. Menschen an Ohrgeräuschen. Die Kenntnisse zur Ätiologie und Pathophysiologie des Tinnitus sind - trotz vielerlei Erklärungsversuche - weiterhin beschränkt [34]; ähnliches gilt für die Behandlung dieses Leidens [36]. Einigen Medikamenten wird eine mögliche Rolle an der Entstehung des Tinnitus zugeschrieben [6]. Darunter befinden sich nichtsteroidale Analgetika (Acetylsalicylsäure [21] u.a.), welche von Patienten mit Gesichtsschmerzen häufig in großen Mengen konsumiert werden, und trizyklische Antidepressiva, die bei Patienten mit persistierenden muskulären MAPBeschwerden häufig im Rahmen eines Behandlungsprogramms verschrieben werden [45].

Obwohl in einigen Untersuchungen eine sehr geringe Prävalenz von Tinnitus bei gleichzeitig vorhandenen MAP-Symptomen gefunden wurde, liegen Hinweise dafür vor, daß MAP-Patienten häufiger von Ohrgeräuschen betroffen sind als Personen ohne MAP (Tabelle 1). In unserer eigenen Klinik geben rund 2 von 3 Patienten an, Ohrgeräusche $\mathrm{zu}$ vernehmen. Eine Analyse von 275 unserer Patientenfragebögen ergab, daß die Frage nach der Anwesenheit von Ohrgeräuschen („Do you have noise or ringing in your ear?") von 185 Personen (67,3\%) bejaht und von 90 Individuen $(32,7 \%)$ verneint wurde ${ }^{1}$. Alle Patienten waren zum Zwecke der Evaluation und Behandlung von Gesichtsschmerzen an unsere Klinik überwiesen worden, und mehr als $95 \%$ von ihnen wiesen eine MAP auf.

Eine methodische Unzulänglichkeit der überwiegenden Zahl der bislang veröffentlichten Studien zur Prävalenz von Ohrsymptomen bei MAPPatienten besteht in dem Fehlen von Vergleichsgruppen. Die Untersuchung von Chole und Parker [7], bei der eine MAP-Gruppe mit 2 Kontrollgruppen verglichen wurden, ist in dieser Hinsicht eine Ausnahme. Die erste Kontrollgruppe umfaßte 326 Patienten, die zur einem routinemäßigen Arztbesuch erschienen; Tinnitus wurde von $13,8 \%$ der Patienten angegeben. Die 2. Kontrollgruppe bestand aus 365 Personen, die sich einer routinemäßigen Zahnreinigung unterzogen; hier betrug der Anteil der Individuen mit Tinnitus 32,5\%. Bei den MAP-Patienten $(n=338)$ war Tinnitus demgegenüber in $59 \%$ der Fälle vorhanden. Obwohl auch diese Studie zeigte, daß Tinnitus bei MAP-Patienten deutlich häufiger vorhanden war, ist bei der Interpretation der Resultate zu beachten, daß aufgrund der gewählten Selektionskriterien der Autoren sämtliche Patienten auch unter Ohrenschmerzen litten. Aus diesem Grunde bezieht sich die in dieser Untersuchung gefundene Prävalenz für Tinnitus auf MAP-Patienten mit gleichzeitiger Otalgie. Chole und Parker wiesen vorsichtshalber darauf in, daß aus ihren Beobachtungen keine Kausalbeziehung zwischen MAPSymptomen und Tinnitus abzuleiten sei [7].

Interessant sind Berichte, nach denen sich bei manchen an Tinnitus leidenden MAP-Patienten die Intensität der Ohrgeräusche durch gegenseitiges Aneinanderpressen der oberen und unteren Zahnreihen, Bewegungen des Unterkiefers oder manuellen Druck auf die Kiefergelenke beeinflussen (in der Regel verstärken) lassen [34, 49]. Einige

\footnotetext{
${ }^{1}$ Die Erfassung dieser Daten wurde von der Ethikkommission der University of Michigan genehmigt (Institutional Review Board for the Use of Human Subjects in Research, Health II, \#1127, 11.2.1997)
}

häufig in der Literatur geäußerten möglichen Erklärungsversuche für das gleichzeitige Auftreten von MAP und Tinnitus seien erwähnt.

Myrhaug [27], und später Arlen [1], versuchten, Tinnitus und andere Symptome (fluktuierende Hörfähigkeit, Vertigo, Wattegefühl im Ohr) bei negativem otologischem Befund über die gemeinsame Innervation der Kaumuskeln, des M. tensor veli palatini und des $M$. tensor tympani durch den N. trigeminus zu erklären. Nach Myrhaugs Vorstellung soll eine vermehrte Aktivität der Kaumuskeln beim Bruxismus über „Reflexkontraktionen“ der intratympalen Muskeln (M. tensor tympani sowie der vom $\mathrm{N}$. facialis innervierte $M$. stapedius) zu den genannten Symptomen führen (sog. „otodentales Syndrom"). Arlen, der die Bezeichnung „otomandibuläres Syndrom“ wählte, sprach in diesem Zusammenhang von einer neuromuskulären Dysfunktion [1]. Die Meinung darüber, ob ein solcher funktioneller Mechanismus möglich ist, geht auseinander [6, 7]. Die ebenfalls von Myrhaug [26] vertretene Hypothese, Ohrsymptome könnten durch Malokklusion der Zähne hervorgerufen worden sein, wird heute weitgehend abgelehnt [6].

Ein anderer Erklärungsversuch stützt sich auf eine bei menschlichen Feten mehrfach beschriebene ligamentäre Verbindung, die durch die Fissura petrotympanica zieht und vom Discus articularis zum Malleus verläuft. Je nach Autor wurde diese Struktur als „diskomalleoläres Band“ oder „tiny ligament“ bezeichnet (Übersicht bei [47]). Da auch bei einigen Erwachsenen die präparatorische Darstellung dieses mit Hilfe von Computertomogrammen nicht darstellbaren Ligaments gelang, wurde seine mögliche Rolle für das Zustandekommen von Ohrgeräuschen [9] diskutiert. Wichmann [52] schließlich spekulierte, daß evtl. ein Zusammenhang zwischen dem Vorliegen von lauten Knackgeräuschen in den Kiefergelenken und der Häufigkeit des Auftretens von Ohrgeräuschen bestehen könnte.

Vernon et al. [49] identifizierten in einer retrospektiven Analyse aus dem zum damaligen Zeitpunkt 1002 Tinnituspatienten umfassenden, im Jahre 1982 begonnenen „Tinnitus Data Registry“ des „Oregon Hearing Research Center" 69 Personen (6,9\%), die zu- 
gleich MAP des Kausystem aufwiesen. Nach Analyse der Patientenbefunde konnten die Autoren keine Variable ausmachen, durch die sich diese 69 Individuen von den 860 Tinnituspatienten der Vergleichsgruppe zweifelsfrei unterschieden.

Die therapeutischen Möglichkeiten bei Vorliegen eines Tinnitus sind trotz einer Vielzahl verschiedener Behandlungsansätze „oft gleich Null“ [20]. Dennoch sind in der (zahn)medizinischen Literatur immer wieder Beiträge erschienen, in denen berichtet wurde, daß es nach zahnärztlichen Therapiemaßnahmen (z.B. nach Inkorporation von Okklusionsschienen) bei einem Großteil der Patienten zu einem Rückgang oder gar einem Verschwinden von Ohrgeräuschen kam (Übersicht bei [6]). Daher haben verschiedene Autoren $[12,29,39]$ empfohlen, Patienten mit - insbesondere akut aufgetretenem Tinnitus mittels funktionell-zahnärztlichen und physiotherapeutischen Maßnahmen zu behandeln. Nach Auswertung von 17 zwischen 1934 und 1993 erschienenen Studien zum Zusammenhang zwischen MAP und Tinnitus stellten Chan und Reade [6] jedoch fest, daß diese Untersuchungen durch deutliche methodische Mängel gekennzeichnet waren. Sie beobachteten, daß viele Autoren dazu neigten, auf der Grundlage von rein deskriptiven Studien nicht haltbare Verallgemeinerungen zu treffen.

Chole und Parker [7] bemerkten, daß Versuche, Tinnitus mit zahnärztlich-okklusalen Therapiemaßnahmen zu behandeln, auf keiner soliden wissenschaftlichen Grundlage stehen. Bei der Beurteilung der Effektivität von Therapiemaßnahmen bei Tinnitus verdient der Hinweis von Rubinstein [34] Beachtung, daß deutliche spontane Besserungen in der Intensität von Ohrgeräuschen bzw. spontane Remissionen wahrscheinlich häufiger vorkommen, als gemeinhin angenommen wird.

\section{Diskussion}

Ob MAP- und die Ohrsymptome als voneinander unabhänge Phänomene anzusehen sind oder ob ein kausaler Zusammenhang zwischen ihnen besteht, ist weiterhin ungeklärt. Eine Schwierigkeit bei der Erforschung einer etwaigen kausalen Beziehung zwischen
MAP und auralen Symptomen besteht darin, daß beide Beschwerdekomplexe in der Bevölkerung weit verbreitet sind. Daher kann man davon ausgehen, daß bei gleichzeitigem Zusammentreffen von Symptomen im Kausystem und im Ohrbereich diese zumindest bei einem Teil der betroffenen Patienten unabhängig voneinander vorhanden sein können. Zudem leidet nicht jeder MAPPatient unter Ohrenproblemen, und nicht jeder Patient mit Ohrenbeschwerden unbekannter Ursache weist MAPSymptome auf. Da jedoch andererseits MAP verschiedenartige Beschwerdebilder beinhalten, ist es zumindest vorstellbar, daß in bestimmten MAP-Untergruppen, die sich durch definierte anatomische, funktionelle oder pathologische Charakteristika auszeichnen, Ohrsymptome häufiger auftreten. Ein solcher Gedanke wird gestützt durch die jüngste Beobachtung von Ren und Isberg [33], die berichteten, daß von 53 Patienten mit einseitigem Tinnitus und ipsilateraler, arthrographisch verifizierter anteriorer Diskuslage (relativ zum Condylus mandibulae) nur bei drei Individuen auch auf der kontralateralen Seite, die frei von Ohrsymptomen war, eine anteriore Diskusposition festzustellen war. Ferner spekulierten die beiden Autoren, daß bestimmte Patientengruppen nicht allein durch das Auftreten eines einzelnen Symptoms, sondern stattdessen durch einen ganzen Komplex verschiedener Beschwerden gekennzeichnet sind. Nach Vergleich der genannten 53 Patienten mit 82 Personen, die eine einseitige anteriore Diskusposition aufwiesen, aber keine Ohrenbeschwerden angaben, stellten Ren und Isberg fest, daß Patienten mit Tinnitus nicht nur durch eine deutlich größere Schmerzintensität in den Kiefergelenken gekennzeichnet waren als Patienten mit anterior positioniertem Diskus ohne Ohrenbeschwerden, sondern auch bedeutend häufiger andere Symptome, darunter Schwindel, Kopfschmerzen und Nackenschmerzen, nannten [33].

Bevor jedoch ernsthaft untersucht werden kann, ob ein kausaler Zusammenhang zwischen MAP und auralen Symptomen besteht, ist es unabdingbar, daß eine primäre Ätiologie der Ohrenbeschwerden zweifelsfrei ausgeschlossen wurde. Die Befunde einer Studie von Brookes et al. [2] mahnen, die Fest- stellung „MAP mit Ohrsymptomen unbekannter Ätiologie“ nicht zu voreilig zu treffen: Nach Untersuchung von 45 MAP-Patienten mit gleichzeitigen Ohrbeschwerden konnten diese Autoren bei 37 Personen (82\%) neben einer Otalgie vorhandene Ohrsymptome, darunter Tinnitus, Hörverlust und Druckgefühl, direkt auf pathologische $\mathrm{Zu}$ stände im HNO-Bereich, wie Otitis, chronische Rhinosinusitis und Otosklerose, zurückführen; bei vier weiteren Individuen wurde eine primäre Ursache im HNO-Bereich für wahrscheinlich gehalten. Nur bei 4 Patienten war keine direkte HNO-Pathologie festzustellen, wobei 2 dieser Patienten an psychischen Erkrankungen litten. Die Autoren kamen daher zu der Schlußfolgerung, daß wenigstens $91 \%$ der Ohrsymtome (Otalgie unberücksichtigt) nicht mit der MAP in Zusammenhang standen.

Mit Vorbehalt betrachtet werden sollte die Hypothese, Ohrsymptome wie Tinnitus könnten durch eine Verbindung zwischen Kiefergelenk und Paukenhöhle ausgelöst werden. Die in einigen mikroskopischen Kiefergelenkpräparaten nachgewiesene Darstellung einer durchgehenden kanalartigen Verbindung zwischen Kiefergelenk und Paukenhöhle läßt zwar Raum für zweifellos äußerst interessante - Spekulationen [9], zunächst gilt es jedoch zu klären, wie häufig eine solche Verbindung vorkommt und ob das darin befindliche Lig. discomalleolare funktionsfähig ist, so daß ein Zug an dieser Struktur, z.B. bei einer Vorwärtsbewegung des Discus articularis im Zuge der Kieferöffnung, die von den Patienten geäußerten Ohrsymptome hervorrufen kann. Aufgrund der fehlenden Darstellbarkeit dieses Ligaments im CT [9] oder MRT ist eine direkte Überprüfung einer solchen Hypothese am Lebenden derzeit nicht möglich. Bei MAP-Patienten mit anteriorer Diskuslage scheint Tinnitus allerdings nicht häufiger vorzukommen als bei MAP-Patienten mit lehrbuchmäßiger Diskusposition [16]. $\mathrm{Ob}$ das Lig. discomalleolare in irgendeiner Weise an dem Auftreten von Ohrgeräuschen beteiligt ist, ist zum gegenwärtigen Zeitpunkt unbekannt. Nach den anatomischen Studien von Schmolke [37] kommt dem diskomalleolären Ligament keine funktionelle Bedeutung zu. 


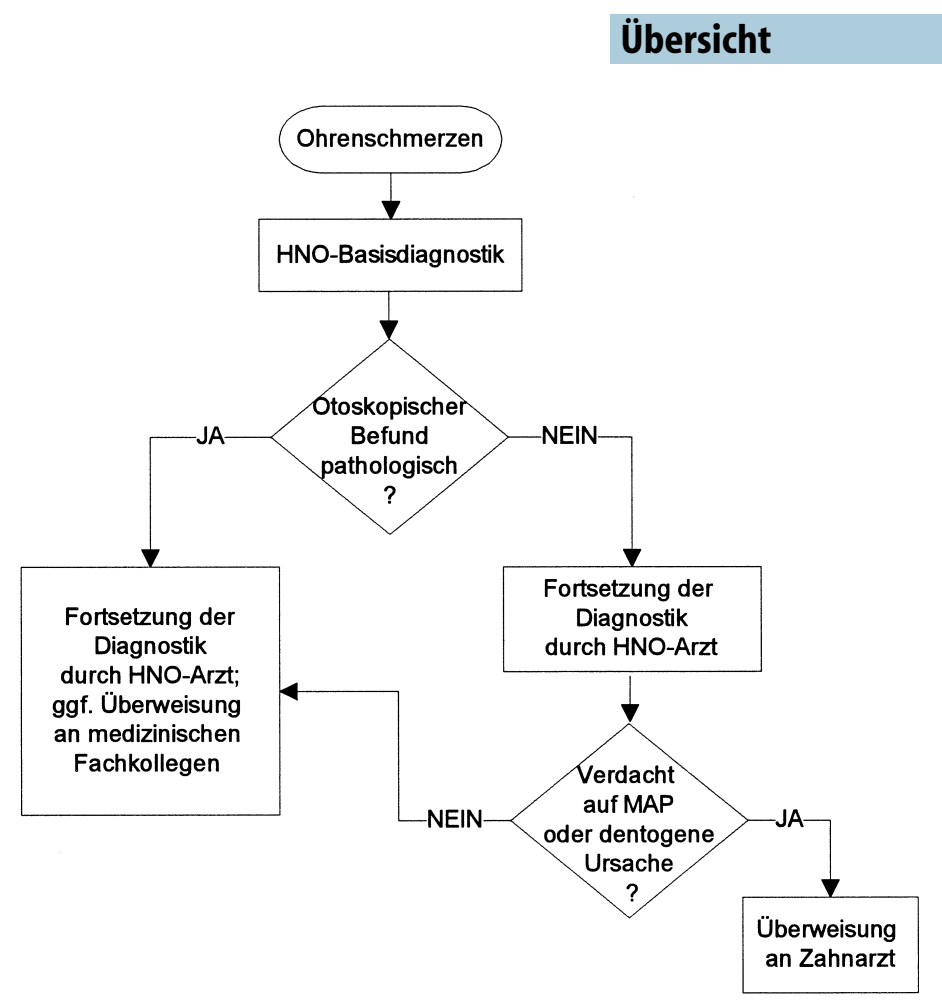

Abb. $1 \Delta$ Klinischer Algorithmus zum diagnostischen Vorgehen bei Patienten mit Ohrenschmerzen und damit möglicherweise in Zusammenhang stehenden Beschwerden im Kausystem (MAP Myoarthropathie)

Die Beurteilung, ob nach erfolgter Behandlung eine Besserung vorhandener Beschwerden eingetreten ist, hängt bei subjektiven Symptomen wie Schmerz oder Tinnitus allein von den Angaben der Patienten ab. In einer prospektiven Studie mit 21 MAP-Patienten gingen Henderson et al. [16] der Frage nach, wie weit Patientenangaben zu eingetretenen Veränderungen im Hörvermögen mit den in audiometrischen Tests erhaltenen „objektiven“ Ergebnissen übereinstimmen. Vor und nach der Behandlung (neun MAP-Patienten wurden konservativ behandelt, bei 13 MAPPatienten wurde eine bilaterale Kiefergelenkarthroskopie durchgeführt) wurden die Patienten gebeten, auf jeweils $100 \mathrm{~mm}$ langen visuellen Analogskalen u.a. das Ausmaß ihres Hörverlusts einzuschätzen. Henderson et al. stellten fest, daß die Patientenangaben und die mit Hilfe der Audiometrie erhaltenen Ergebnisse nicht miteinander korrelierten. So gaben einige Patienten nach $\mathrm{Ab}$ schluß der Therapie eine deutliche Besserung der Hörfähigkeit an, ohne daß sich der subjektiv wahrgenommene Unterschied in den vor und nach der Behandlung ausgeführten audiometrischen Messungen widerspiegelte, während andere Patienten von keinen Ver- änderungen in der Hörfähigkeit berichteten, obwohl solche objektiv vorhanden waren [16]. Diese Ergebnisse zeigen, daß subjektive Beschwerden oft in keinem engen Zusammenhang mit physiologischen Meßresultaten stehen. Dieses Phänomen wird in ähnlicher Form in vielen Bereichen der Medizin angetroffen, wie z.B. in der Radiologie, wo mit bildgebenden Verfahren gewonnene „objektive“ Befunde oft nicht oder nur schwach mit von Patienten angegebenen Symptomen korrelieren.

\section{Fazit für die Praxis}

Wenn die HNO-ärztliche Untersuchung von Patienten mit Otalgie keine Hinweise auf eine Ohrenerkrankung ergab, der HNOArzt aber den Verdacht hat, die Ursache der vom Patienten angegebenen $\mathrm{Be}$ schwerden könnte im Kiefergelenkbereich oder anderen Strukturen des stomatognathen Systems lokalisiert sein, sollte zwecks Abklärung eine Überweisung an einen Zahnarzt mit Erfahrung auf dem Ge-



Abb. $2 \Delta$ Klinischer Algorithmus zum diagnostischen Vorgehen bei Patienten mit Tinnitus und möglichem Vorliegen von Myoarthropathien des Kausystems (MAP Myoarthropathie) 
biet der MAP des Kausystems erfolgen (Abb. 1)

Da bislang keine kontrollierten klinischen Studien vorliegen, die die Wirksamkeit einer Behandlung des Tinnitus durch funktionell-zahnärztliche und physiotherapeutische Maßnahmen belegen, sollte in diesen Fällen eine entsprechende Empfehlung mit der notwendigen Vorsicht ausgesprochen werden.Zweifelsohne ist eine Überweisung an einen Zahnarzt in denjenigen Fällen sinnvoll, in denen der Patient neben und unabhängig von Ohrgeräuschen an Schmerzen im Bereich der Kiefergelenke und Kaumuskeln leidet oder deutliche Einschränkungen der Unterkieferbeweglichkeit aufweist (Abb. 2).

Berichtet umgekehrt ein Patient in der zahnärztlichen Praxis von Ohrenschmerzen oder Ohrgeräuschen, so ist durch einen HNO-Arzt abzuklären, ob die Beschwerden durch Entzündungen oder andere im HNO-Bereich lokalisierte Prozesse bedingt sind. Der Zahnarzt sollte daran denken, daß Otalgien unter anderem durch akute oder chronische Prozesse im orofazialen Bereich (z.B.Zähne, Kiefergelenke, tiefe Portion des M. masseter) hervorgerufen werden können [31]. Daher sind diese Strukturen als mögliche Ursachen von Schmerzen im Bereich der Ohren auszuschließen.

Behauptungen einer kausalen Beziehung zwischen MAP und Ohrenbeschwerden beruhen weiterhin auf reiner Spekulation. Die Frage, ob zahnärztliche und nichtzahnärztliche Therapiemethoden eine spezifische Wirkung für die Behandlung von Ohrenprobleme bei MAP-Patienten aufweisen, kann nur mit Hilfe von prospektiven, kontrollierten und randomisierten Studien beantwortet werden. Solche Untersuchungen sind bislang nicht vorhanden.

Der Autor möchte sich bei Herrn Prof. Dr. Christian Stohler für die Durchsicht des Manuskripts herzlich bedanken.

\section{Literatur}

1. Arlen $\mathrm{H}$ (1977) The otomandibular syndrome: a new concept. Ear Nose Throat J 56:60-62

2. Brookes GB, Maw AR, Coleman MJ (1980) 'Costen's syndrome' - correlation or coincidence: a review of 45 patients with temporomandibular joint dysfunction, otalgia and other aural symptoms. Clin Otolaryngol 5:23-26
3. Bruck A (1895) Ueber eine unter dem Bilde einer Ohrenerkrankung verlaufende Neurose des Kiefergelenks. Dtsch Med Wochenschr 21:530-532

4. Bush FM (1987) Tinnitus and otalgia in temporomandibular disorders. J Prosthet Dent 58:495-498

5. Carlsson GE, Kopp S, Wedel A (1982) Analysis of background variables in 350 patients with TMJ disorders as reported in self administered questionnaire. Comm Dent Oral Epidemiol 10:47-51

6. Chan SW, Reade PC (1994) Tinnitus and temporomandibular pain-dysfunction disorder. Clin Otolaryngol 19:270-380

7. Chole RA, Parker WS (1992) Tinnitus and vertigo in patients with temporomandibular disorder.

Arch Otolaryngol Head Neck Surg 118:817-821

8. Costen JB (1934) A syndrome of ear and sinus symptoms dependent upon disturbed function of the temporomandibular joint. Ann Otol Rhinol Laryngol 43:1-15

9. Eibl K, Solar P, Ulm C, Slavicek R (1994) Die Knochenkanäle der tympanomandibulären und discomalleolären Ligamente Nachweis und Lokalisation. Z Stomatol [Suppl] 6:27-28

10. Gelb H, Bernstein I (1983) Clinical evaluation of two hundred patients with temporomandibular joint syndrome. J Prosthet Dent 49:234-243

11. Gelb H, Calderone JP, Gross SM, Kantor ME (1967) The role of the dentist and the otolaryngologist in evaluating temporomandibular joint syndromes. J Prosthet Dent 18:497-503

12. Gelb H, Gelb ML, Wagner ML (1997) The relationship of tinnitus to craniocervical mandibular disorders. Cranio 15:136-143

13. Goodfriend DJ (1947) Deafness, tinnitus, vertigo and neuralgia. Arch Otolaryngol 46:1-35

14. Gross AJ, Rivera-Morales WC, Gale EN (1988) A prevalence study of symptoms associated with TM disorders. J Craniomandib Disord 2:191-195

15. Hankey GT (1957) Some observations on Costen's mandibular snydrome: including a review of $\mathbf{1 0 0}$ out of $\mathbf{5 0 0}$ cases. Proc R Soc Med 51:225-232

16. Henderson DH, Cooper JC Jr., Bryan GW, Van Sickels JE (1992) Otologic complaints in temporomandibular joint syndrome. Arch Otolaryngol Head Neck Surg 118:1208-1213

17. Johansson AS, Isberg A, Isacsson G (1990) A radiographic and histologic study of the topographic relations in the temporomandibular joint region: implications for a nerve entrapment mechanism. J Oral Maxillofac Surg 48:953-961

18. Keersmaekers K, De Boever JA, Van Den Berghe $L$ (1996) Otalgia in patients with temporomandibular joint disorders. J Prosthet Dent 75:72-76
19. Kempf HG, Roller R, Mühlbradt L (1993) Über die Beziehung von Innenohrstörungen und Kiefergelenkserkrankungen. HNO 41:7-10

20. Lehnhardt E (1992) HNO-Heilkunde für Zahnmediziner, 2. Aufl. Thieme, Stuttgart New York

21. Lenarz T (1996) Acetylsalizylsäureinduzierter Tinnitus. HNO 44:272

22. Lobbezoo F, Lavigne GJ (1997) Veroorzaakt bruxisme klachten van TMD? Ned Tijdschr Tandheelkd 104:24

23. McNamara JA Jr, Türp JC (1997) Orthodontic treatment and temporomandibular disorders: is there a relationship? Part I. Clinical studies. Fortschr Kieferorthop 58:74-89

24. McNeill C (1997) History and evolution of TMD concepts. Oral Surg Oral Med Oral Pathol Oral Radiol Endod 83:51-60

25. Morgan DH (1975) Surgical correction of temporomandibular joint arthritis. J Oral Surg 33:766-773

26. Myrhaug $\mathrm{H}$ (1964) The incidence of ear symptoms in cases of malocclusion and temporo-mandibular joint disturbances. Br J Oral Surg 2:28-32

27. Myrhaug H (1969) Parafunktionen im Kauapparat als Ursache eines otodentalen Syndroms. 1. Quintessenz 20:117-121

28. National Institutes of Health (1996) National Institutes of Health Technology Assessment Conference Statement: management of temporomandibular disorders. J Am Dent Assoc 127:1595-1603

29. Neuhauser W (1992) Tinnitus als zahnärztliches Problem. Kiefergelenksaffektionen und Bruxismus. Diagnostische und therapeutische Möglichkeiten. In:Goebel G (Hrsg) Ohrgeräusche.Psychosomatische Aspekte des komplexen Tinnitus.Vorkommen, Auswirkungen, Diagnostik und Therapie. Quintessenz, München, S 245-269

30. Ohrbach R, Stohler CS (1992) Review of the literature: current diagnostic systems. J Craniomand Disord Facial Oral Pain 6:307-317

31. Okeson JP (1996) Orofacial pain. Guidelines for assessment, diagnosis, and management. Quintessence, Chicago

32. Posselt U (1971) The temporomandibular joint syndrome and occlusion. J Prosthet Dent 25:432-438

33. Ren YF, Isberg A (1995) Tinnitus in patients with temporomandibular joint internal derangement. Cranio 13:75-80

34. Rubinstein B (1993) Tinnitus and craniomandibular disorders - is there a link? Swed Dent J [Suppl] 95:1-46

35. Rubinstein B, Carlsson GE (1987) Effects of stomatognathic treatment on tinnitus: a retrospective study. Cranio 5:254-259

36. Rudack C, Hillebrandt M, Wagenmann M, Hauser U (1997) Tinnitusbehandlung mit Lidocain? HNO 45:69-73 
37. Schmolke C (1994) The relationship between the temporomandibular joint capsule, articular disc and jaw muscles. J Anat 184:335-345

38. Schulte W (1981) Myoarthropathien. Epidemiologische Gesichtspunke, analytische und therapeutische Ergebnisse. Dtsch Zahnärztl Z 36:343-353

39. Schulte W (1993) Hörstörungen durch Myoarthropathien des Kiefergelenkes. HNO 41:12

40. Seligman DA, Pullinger AG (1991) The role of functional occlusal relationships in temporomandibular disorders: a review. $\mathrm{J}$ Carniomandib Disord Facial Oral Pain 5:265-279

41. Seligman DA, Pullinger AG (1991) The role of intercuspal occlusal relationships in temporomandibular disorders: a review. $J$ Craniomandib Disord Facial Oral Pain 5:96-106

42. Sicher H (1948) Temporomandibular articulation in mandibular overclosure. J Am Med Assoc 36:131-139

43. Slavkin HC (1996) A lifetime of motion: temporomandibular joints. J Am Dent Assoc 127:1093-1098

44. Turner JA, Deyo RA, Loeser JD, Von Korff M, Fordyce WE (1994) The importance of placebo effects in pain treatment and research. J Am Med Assoc 271:1609-1614

45. Türp JC (1997) Myoarthropathien des Kausystems - mehr als nur ein zahnmedizinisches Problem. Dtsch Med Wochenschr 122:483-487

46. Türp JC, Gobetti JP (1996) Trigeminal neuralgia versus atypical facial pain. A review of the literature and case report. Oral Surg Med Oral Pathol Oral Radiol Endod 81:424-432

47. Türp JC, Obrez A, Radlanski R (1997) Anatomie und Ontogenese des menschlichen Kiefergelenks. In: Alt KW, Türp JC (Hrsg) Die Evolution der Zähne - Phylognie, Ontogenie, Variation. Quintessenz, Berlin, S 647-675

48. Türp JC, Strub JR (1996) Gelenkgeräusche aus der Sicht der Orthopädie - eine Fragebogenstudie unter Lehrstuhlinhabern. Dtsch Zahnärztl Z 51:345-349

49. Vernon J, Griest S, Press L (1992) Attributes of tinnitus that may predict temporomandibular joint dysfunction. Cranio 10:282-287

50. Vero D (1995) Facial pain in association with temporo-mandibular joint dysfunction. J Laryngol 0tol 79:707-723

51. Wedel A, Carlsson GE (1985) Factors influencing the outcome of treatment in patients referred to a temporomandibular joint clinic. J Prostet Dent 54:420-426

52. Wichmann M (1994) Die Schallpegel bei Kiefergelenkgeräuschen. Dtsch Zahnärztl Z 49:893-895

\section{K.-B. Hüttenbrink \\ Middle Ear Mechanics and \\ Research and Otosurgery}

Dresden: Univ. of Technology, 1997.259 S., 208 Abb., 24 Tab., (ISBN 3-86005-193-8), geb., Preis entspricht den Portogebühren

Das von Hüttenbrink herausgegebene Buch über die Mittelohrmechanik in Forschung und Ohrchirurgie beinhaltet eine Zusammenstellung der Vorträge, die auf dem Internationalen Workshop über Mittelohrmechanik in Forschung und Ohrchirurgie am 19.-22. September 1996 in Dresden abgehalten wurden. Das vorliegende Buch spiegelt in seiner klaren Darstellungsart den aktuellen Kenntnisstand über die Funktion des Mittelohres wider.

In übersichtlicher

Zusammenstellung gliedert sich das Buch in 3 Themenkomplexe.

1. Das normale Mittelohr,

2. die Rekonstruktion des Mittelohres mit den Themenschwerpunkten Biomaterialien und mechanische Grundlagen und

3. der Einfluß des Mittelohres auf die otoakustischen Emissionen.

In bemerkenswerter Art und Weise ist es dem Herausgeber gelungen, international herausragende Referenten für die Beiträge zur Messung der physiologischen und pathophysiologischen Funktionen des Mittelohres mittels Laserdopplervibrometrie und computersimulierter Animation durch Finite-Elemente-Modelle zu gewinnen. Be- sonderen Wert erzielt das vorliegende Buch durch die Synthese der Erkenntnisse aus theoretischen Hörforschungseinrichtungen mit den Erfahrungen der klinisch tätigen Otologen. So wird das Schwingungsverhalten des Trommelfells analysiert und der Versuch unternommen, aus diesen Erkenntnissen die Möglichkeiten der Rekonstruktion des Trommelfells zu verbessern oder das Design einer idealen Mittelohrprothese zu optimieren.

Besonders interessantwird das Buch durch die Wiedergabe der Diskussionsbeiträge. Dabei wurden die sich nach den Vorträgen ergebenden Fragen und die dazugehörigen Antworten der Referenten abgedruckt. Dadurch wird dem Leser in geeigneter Weise die Lebendigkeit der Diskussion vermittelt.

Das vorliegende Buch zeichnet sich ferner durch eine Reichhaltigkeit der Abbildungen aus. Dem Herausgeber ist es gelungen, die theoretischen Aspekte und klinischen Erfahrungen der Forscher und Ohrchirurgen auf ideale Weise zusammenzufügen und so für ein besseres Verständnis der Mittelohrmechanik und der Entwicklung verbesserter Rekonstruktionsmaßnahmen in der Ohrchirurgie nutzbar zu machen. Unterstützt durch die Fa. Kurz, Dusslingen, konnte eine attraktive Gestaltung des Buches realisiert werden. Das von K.-B. Hüttenbrink herausgegebene Buch zur Mittelohrmechanik spiegelt den sog. State of the Art wider und sollte in keiner Fachbibliothek fehlen.

M.M.Maaßen (Tübingen) 